\title{
Accurate Closed-form Estimation of Local Affine Transformations Consistent with the Epipolar Geometry
}

\author{
Daniel Barath, Levente Hajder \\ \{barath.daniel,hajder.levente\}@sztaki.mta.hu \\ Jiri Matas \\ matas@cmp.felk.cvut.cz
}

\author{
MTA SZTAKI \\ Budapest, Hungary \\ CMP, Czech Technical University \\ Prague, Czech Republic
}

A novel method is proposed for accurate estimation of local affine transformations for a pair of images satisfying the epipolar constraint. The method returns the closest, in least squares sense, affine transformation to an initial estimate consistent with the fundamental matrix.

The contributions of the paper: (i) the introduction of two novel constraints for a local affine transformation making it consistent with the fundamental matrix, and (ii) a method estimating an EG- $L_{2}$-Optimal affinity - transformation which is consistent with the epipolar geometry (EG) -, by enforcing the proposed constraints.

An affine correspondence consists of a point pair $\mathbf{p}_{1}, \mathbf{p}_{2}$ and a local affine transformation A mapping the neighborhood of the points.

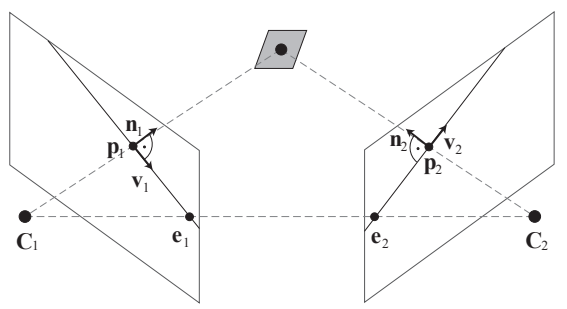

The constraints state that the $2 \times 2$ matrix $\mathbf{A}$ transforms the normal $\mathbf{n}_{1}$ of the epipolar line related to point $\mathbf{p}_{1}$ into $\beta \mathbf{n}_{2}$, where $\mathbf{n}_{2}$ is the normal of the epipolar line related to point $\mathbf{p}_{2}$ and $\beta \in \mathbb{R}$ is a scalar. This statement is equivalent to $\mathbf{n}_{1} \mathbf{A}^{-T}=$ $\beta \mathbf{n}_{2}$. It is proven as well that $\beta$ is determined by the epipolar geometry.

The method requires an affine correspondence $\mathbf{p}_{1}, \mathbf{p}_{2}, \mathbf{A}^{\prime}$, i.e. estimated by an affine-covariant detector. The points $\mathbf{p}_{1}$ and $\mathbf{p}_{2}$ are optimally be corrected w.r.t. the epipolar geometry, in least squares sense, by the method of [4]. The proposed technique corrects $\mathbf{A}^{\prime}$ by simultaneously minimizing $\left\|\mathbf{A}-\mathbf{A}^{\prime}\right\|_{F}^{2}$ and enforcing the introduced constraints using a closed-form approach. It is proven that $\left\|\mathbf{A}-\mathbf{A}^{\prime}\right\|_{F}^{2}$ has both geometric and algebraic interpretations.

The processing time of the method is $\approx 0.04 \mathrm{~ms}$ in $\mathrm{C}++$.

Evaluation. The method is validated on synthetic data and publicly available benchmarks. The corrected affinities are always more accurate than the output of the affine-covariant detector. As a side-effect, the detectors are compared - the most accurate is the Hessian-Affine augmented by view-synthesis a la ASIFT.

Conclusions. The algorithm has negligible time demand and always makes the input affinities more accurate. In problems involving local affine transformations in rigid scenes, the proposed method should always be used.
Application 1. Using the proposed results the detection and segmentation of multiple planes becomes more accurate [1].
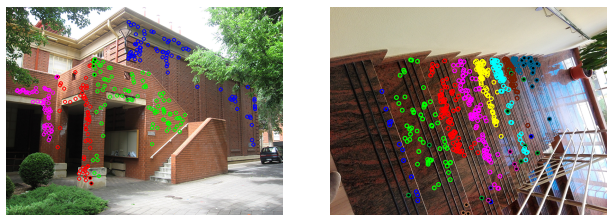

$\overline{\text { Application 2. Using equation } \mathbf{n}_{1} \mathbf{A}^{-T}=\beta \mathbf{n}_{2} \text { the fundamental }}$ matrix is estimable from two affine correspondences.
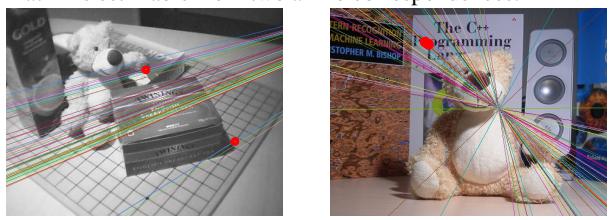

Application 3. Surface normal estimation benefiting from precise affine correspondences [2].
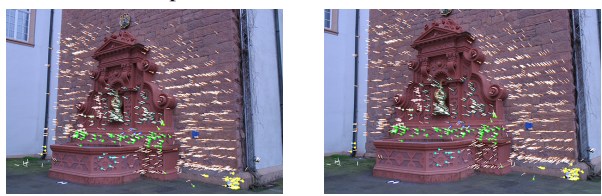

Application 4. Precise affine correspondences significantly improve camera calibration as well as 3D reconstruction [3].
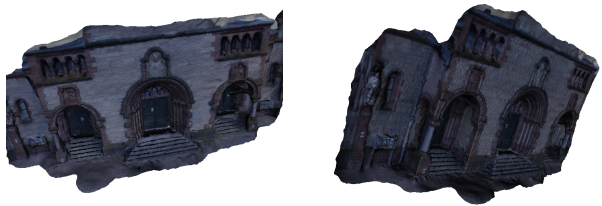

Application 5. In the paper, we use the method to compare the geometric precision of affine-covariant feature detectors.

[1] D. Barath, J. Matas, and L. Hajder. Multi-H: Efficient recovery of tangent planes in stereo images. In $B M V C$, 2016.

[2] D. Barath, J. Molnar, and L. Hajder. Novel methods for estimating surface normals from affine transformations. In VISIGRAPP Selected Papers, 2016.

[3] I. Eichhardt and L. Hajder. Improvement of camera calibration using surface normals. In ICPR, 2016.

[4] R. I. Hartley and P. Sturm. Triangulation. CVIU, 1997. 